\title{
Psychological Impact of Preconception Counseling: Assessment of Anxiety before and during Pregnancy
}

\author{
Sabina de Weerd ${ }^{a}$ Akke K. van der Bij ${ }^{b}$ Jozé C.C. Braspenning ${ }^{b}$ \\ Rolf J.L.M. Cikot ${ }^{a}$ Didi D.M. Braat ${ }^{a}$ Eric A.P. Steegers ${ }^{c}$ \\ ${ }^{a}$ Department of Obstetrics and Gynecology, University Medical Center Nijmegen, ${ }^{b}$ Center for Quality of Care \\ Research, University of Nijmegen, and 'Department of Obstetrics and Gynecology, University Hospital Rotterdam, \\ The Netherlands
}

\section{Key Words}

Screening · Anxiety $\cdot$ Preconception care $\cdot$ Preconception counseling · Pregnancy · Spielberger State-Trait Anxiety Inventory

\begin{abstract}
Objective: Assessment of anxiety levels in women and men before and after preconception counseling and during the first trimester of pregnancy. Methods: Couples were recruited from the fertility clinic of the University Medical Center Nijmegen, the Netherlands. Anxiety was assessed using the 40-item Spielberger State-Trait Anxiety Inventory (STAI). Results: 53 women and 51 men (74\%) completed the STAI both before and after counseling. Anxiety levels did not change significantly after counseling or during the first trimester of pregnancy. 83.4\% would recommend preconception counseling to others. Conclusion: Preconception counseling is valued by the majority of women and men and does not lead to adverse psychological effects.
\end{abstract}

Copyright ( $\subset 2002$ S. Karger AG, Basel

\section{Introduction}

Preconception care anticipates obstetric, genetic, and other health problems that may arise in couples planning a pregnancy [1]. Identification of risk factors through assessment of many different aspects of health, lifestyle and family history before conception provides a unique opportunity to address potentially harmful situations that may adversely affect pregnancy outcome. Possible risks may be signaled, couples can be educated about actions they can take to promote desirable outcome, and often interventions can be initiated. However, many conditions exist that cannot be altered. This especially applies to cases where risks related to family history are identified, where interventions are often not possible and couples face difficult decisions. Although preconception care and subsequent counseling have clear benefits that may contribute to better health of mother and child [1], the possible harm caused by this form of prevention also needs to be considered.

A frequent concern is the psychological impact of screening. The psychological harm that screening programs may cause includes anxiety over the results, and implications and consequences of being labeled as 'at

Dr. E.A.P. Steegers, Associate Professor in Obstetric

Department of Obstetrics and Gynecology

University Hospital Rotterdam, PO Box 2040

NL-3000 CA Rotterdam (The Netherlands)

Tel. +31 104633632, Fax +31 104635826
Fax + 41613061234

E-Mail karger@karger.ch www. karger.com

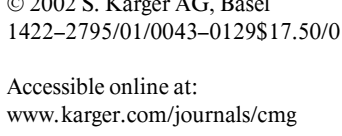


risk' [2]. Various studies report raised levels of anxiety amongst those with positive results or risks identified through various screening programs, and illustrate that the receipt of a negative result is not always reassuring [3]. The possible adverse effects of screening should be evaluated before the widespread implementation of a population-based preventive program such as the provision of preconception care to all women planning to conceive. Couples may not want to know if their health state or family history could harm their future offspring. Whether counseling is appreciated, and whether and to what extend anxiety is induced by the consultation, are important preliminary issues that need to be evaluated.

In this study, the effect of preconception screening and subsequent counseling on anxiety levels was studied in couples recruited from a fertility clinic. It could be that a possible effect of counseling is not seen immediately, but becomes evident during pregnancy, at which time couples could start to worry about issues discussed at the preconception consultation or experience increasing anxiety because of their particularly 'precious' pregnancy after a history of infertility [4]. Therefore, anxiety levels were evaluated again among those who became pregnant.

\section{Methods}

Between September 1997 and April 1999, 121 couples attending the fertility clinic of the University Medical Center Nijmegen, the Netherlands, were informed by a gynecologist or resident about the study on the desirability and effect of preconception counseling. Most Dutch women do not seek care until pregnancy has been established, which poses great difficulty in recruiting a low-risk population preconceptionally. Study participants were recruited from a fertility clinic because all were planning pregnancy, and although they form a selected population, were not expected to differ from women conceiving naturally in terms of preexistent risk factors for adverse pregnancy outcome [5]. The couples received a letter that contained information about purpose and design of the study and invited them to participate. After giving their informed consent, 71 women and 70 men completed the first Spielberger State-Trait Anxiety Inventory (STAI) questionnaire. The Medical Ethical Committee of the University Medical Center Nijmegen approved the study.

A validated Dutch version of the STAI-DY $[6,7]$ was used to detect changes in anxiety levels. This self-evaluation questionnaire is a reliable and sensitive instrument, and one of the most frequently used measures of anxiety in applied psychology research [6,7]. The full version consists of 40 items, with 20 pertaining to stress disposition (trait-anxiety) which is considered to be stable over time, and 20 pertaining to current anxiety levels (state-anxiety), which is sensitive to change. Answers are based on a four-point scale, with total scores ranging from 20 to 80 . Precounseling trait scores were measured for comparison with STAI-trait scores of the Dutch population and to exclude possible bias of the study outcome. Changes in STAI-state scores were considered to reflect the impact of counseling (with a higher postcounseling score indicating an adverse effect and a lower score a reassuring effect). A mean increase in STAI-state score of 11 points among women and 9 points among men was found in the reference population after a stressful situation [6]. In order to detect such a difference in mean STAI-state scores, 52 participants were needed for our study $(\alpha=0.05$; power $=90 \%)$.

Participants were asked to complete the STAI questionnaire for the first time (STAI-1) before their intake visit with a physician at the Obstetrics and Gynecology Department. During this visit, a broad range of possible risk factors for adverse pregnancy outcome was assessed using a Preconceptional Health assessment form [1] and a family history survey. These screening questionnaires were completed beforehand and verified during this consultation. Another appointment was made 4-6 weeks later, allowing time for further investigations or consultation of other experts if necessary, at which time any applicable risk factors and their implications or possible consequences were discussed. General aspects of prepregnancy health care such as smoking cessation, proper nutrition, folic acid supplementation, and a plan for antenatal care were also covered during the counseling session. Couples received the STAI again after this counseling session to be completed at home and returned within 2 weeks after counseling (STAI-2). Nonresponders were encouraged by phone to send back the questionnaires within this period. When pregnancy occurred, couples were asked to complete the survey once more in the first trimester (STAI-3). Several questions specific to anxiety during the current pregnancy and a question on the appreciation of preconception counseling were included in STAI-3.

Mean STAI-state and trait scores before counseling (preintervention) were determined for the entire study population and for the subgroups that completed more than one STAI questionnaire. A paired t-test was performed to assess the effect of counseling on anxiety among participants that completed the STAI both before and after counseling (STAI-1 and 2) and before counseling and during pregnancy (STAI-1 and 3). A frequency table (\%) was made to evaluate the additional pregnancy-specific questions of STAI-3. Statistical significance was defined by $\mathrm{p}<0.05$.

\section{Results}

\section{Population}

Seventy-one women and 70 men completed STAI-1, 56 women and 55 men completed STAI- 2 and 42 men and 42 women completed STAI- 3 . The effect of counseling on anxiety could be evaluated in $74 \%$ of the study population (STAI-1 vs. 2), and a possible long-term effect of counseling (STAI-1 vs. 3 ) was assessed in $57 \%$ of those who became pregnant. Mean age (SD) of the participants was 34.5 (3.9) years. Baseline STAI-state and trait scores are comparable for the three subgroups (table 1) though stress disposition is slightly higher among women who completed both STAI-1 and 3. As only 27 participants completed the STAI at all three points in time, their data were not considered to be representative. 


\section{Effect on Anxiety}

Preconception counseling did not induce anxiety among the study population. No significant change in anxiety levels was seen in men or women after counseling (table 2). STAI-state scores were actually lower during the first trimester of pregnancy as compared to precounseling scores, though this difference is not statistically significant. Although STAI-state scores of the men were lower at all points in time, no significant differences in scores were found between men and women.

Table 1. STAI-scores before counseling

\begin{tabular}{llll}
\hline Population $^{1}$ & $\mathrm{n}$ & STAI-state & STAI-trait \\
\hline Women & & & \\
STAI 1 & 71 & $35.5(10.4)$ & $35.6(8.8)$ \\
STAI 1 and 2 & 53 & $35.2(9.9)$ & $34.6(7.3)$ \\
STAI 1 and 3 & 24 & $36.8(11.9)$ & $37.1(9.7)$ \\
Van der Ploeg [6] & 127 resp. 125 & $38.0(12.8)$ & $39.0(11.1)$ \\
\hline Men & & & \\
STAI 1 & 70 & $33.9(8.0)$ & $32.6(8.4)$ \\
STAI 1 and 2 & 51 & $33.5(6.9)$ & $32.8(7.4)$ \\
STAI 1 and 3 & 24 & $34.7(7.7)$ & $32.8(10.2)$ \\
Van der Ploeg [6] & 126 resp. 124 & $35.8(8.8)$ & $36.5(9.5)$ \\
\hline
\end{tabular}

Figures given are means (SD).

1 Precounseling scores are shown separately for women and men that completed STAI-1 only, STAI 1 and 2 (before and after counseling), and those that completed STAI-1 and 3 (before counseling and during pregnancy).
In the pregnancy-specific items related to stress, included in STAI-3, half of the couples reported feeling concerned when thinking about the baby and the majority $(82 \%)$ worried to some extent about the pregnancy (table 3). However, almost all had confidence in the pregnancy outcome (98.8\%). The question 'Would you recommend preconception counseling to friends and acquaintances trying to conceive?' was answered affirmatively by $83.4 \%$ of couples $(56 \%$ answered yes, and $27.4 \%$ would probably recommend preconception counseling). 14.3\% would probably not recommend preconception counseling, and only one couple answered no.

\section{Discussion}

This is the first study on the psychological impact of preconception counseling in terms of anxiety before and during pregnancy. Though it is undisputed that preconception counseling should be accessible to women at high risk for adverse pregnancy outcome, no consensus has been reached whether low-risk women and their partners should be offered preconception care as well. We feel that the women in our study can overall be considered as being at low-risk for adverse pregnancy outcome, based on a Dutch matched control study on outcome of singleton in vitro fertilizations (IVF) and spontaneously conceived pregnancies. Although significantly more pregnancies ended preterm and a larger number of small for gestational age babies were born in the IVF group, no significant differences in pregnancy complications, perinatal mortal-

Table 2. Change in anxiety scores (STAI-state)

\begin{tabular}{lllll}
\hline & $\begin{array}{l}\text { After counseling } \\
\text { (STAI 1 vs. 2) }\end{array}$ & $\begin{array}{l}\text { p value } \\
\text { paired t-test }\end{array}$ & $\begin{array}{l}\text { During pregnancy } \\
\text { (STAI 1 vs. 3) }\end{array}$ & $\begin{array}{l}\text { p value } \\
\text { paired t-test }\end{array}$ \\
\hline Women & +0.1 & 0.980 & -1.1 & 0.649 \\
Men & -0.8 & 0.373 & -2.2 & 0.091 \\
\hline
\end{tabular}

Table 3. Anxiety specifically related to pregnancy

\begin{tabular}{lccrr}
\hline Question (included with STAI 3) & Not at all & Somewhat & Quite a bit & Very much \\
\hline I worry about my pregnancy & 17.9 & 61.9 & 14.3 & 6.0 \\
I am nervous when I think about the baby & 51.2 & 39.3 & 8.3 & 1.2 \\
I am confident about the outcome of my pregnancy & 1.2 & 15.5 & 53.6 & 29.8 \\
\hline
\end{tabular}

Figures given are percentages. 
ity, or neonatal condition were found in the study carried out at four university hospitals in the Netherlands [5]. In addition, our study group was not a homogeneous IVF population but included women who took longer to conceive without any further problems.

A question often raised is whether participating in a screening program adds to the alarm people already feel about their health [2]. This effect could be induced through expansion of preconception screening and subsequent counseling to a larger target group. Ideally, this issue should be addressed among women who have not needed to consult a gynecologist for any reason, since it is likely that the women in our study are already more aware of various aspects of preconceptional health and wellbeing due to their difficulty in becoming pregnant. However, neither women visiting fertility clinics nor women conceiving naturally are offered routine preconception screening in the Netherlands. In our population, no such adverse effects of counseling (using change in anxiety as an indicator of alarm) were found. One could imagine that this reassuring effect is only short-term and that anxiety levels would rise again once women are actually pregnant, though this was not the case. STAI-state scores were even lower during pregnancy than before counseling. Whether this reflects a long-term reassuring effect of counseling, or is perhaps due to the relief of the (often) long-awaited pregnancy, is unknown. Nevertheless, as only one pregnant couple indicated not having confidence in the pregnancy outcome, it seems likely that most couples were reassured to some extent by the consultation.

No postcounseling data were available for 18 women and 19 men because they did not send back the STAI questionnaires within the 2-week period after counseling. Efforts to collect the forms were ceased after this time period in order to maintain comparable data on the shortterm effect of counseling. Because the baseline anxiety level of the entire study group did not differ significantly from that of the women and men that did complete the second STAI questionnaire (table 1), it seems that the obtained results are representative.

One could question whether couples recruited from a fertility clinic are representative of the general population in terms of current anxiety and stress disposition because of their infertility. To address this issue, we compared precounseling STAI-state and trait scores of our study group to reference values found in a general Dutch population of women and men between 16 and 40 years of age [6]. Both STAI-trait and state scores were actually lower in our study group (table 1), so that we could infer that women and men in our clinical setting are not more dis- posed to anxiety than the population at large. It also seems unlikely that the possible anticipation of screening elevates anxiety before consultation, otherwise higher precounseling STAI-state scores would be expected. Furthermore, a recent American study showed that pregnant IVF women are similar psychologically to women who become pregnant naturally in terms of anxiety (as well as depression and self-esteem) at 12 and 28 weeks gestational age [4].

A study by Rose et al. [8] on the feasibility and acceptability of genetic counseling in primary care also evaluated anxiety levels before and after consultation and again after 12 weeks using the short version of the STAI. A significant reduction in anxiety was found immediately after consultation, and returned to initial anxiety levels after 12 weeks. They conclude that counseling results in a shortterm reduction in anxiety, with no long-term adverse effects. This difference with our results in postcounseling anxiety is most probably attributable to the difference in study population. Psychology research has shown that screening generally provides some certainty for those already identified as being at increased risk, regardless of the result [3], which could explain the reduction in anxiety found after counseling in their population and not among women attending our fertility clinic. In their study, couples were invited by letter to attend a clinic for genetic counseling, and although one-fifth came out of general interest, many attended the consultation because they were aware of diseases that ran in the family and were (or felt) at higher risk for adverse pregnancy outcome. This is in contrast to the specific problem for which women attended our clinic, namely to seek help in becoming pregnant after an unsuccessful 1-year period.

Preconception counseling, currently only offered to women at high risk for adverse pregnancy outcome in the Netherlands, is a largely unknown health care service among the general population and usually not actively sought by couples planning pregnancy. Although we only have one indicator, it seems that most couples appreciated counseling considering that the majority would recommend it to others.

Despite the opportunities for prevention and education offered through preconception counseling, structural implementation and accessibility to all couples planning pregnancy remains debated. Our results, demonstrating acceptability and a lack of adverse psychological effects of preconception counseling, should be taken into account during further considerations of expanding preconception care to a larger target population.
$132 \quad$ Community Genet 2001;4:129-133 de Weerd/van der Bij/Braspenning/Cikot/ Braat/Steegers 


\section{References}

1 Cefalo RC, Moos MK: Preconceptional health care. A practical guide. St. Louis, Mosby-Year Book, 1995.

2 Marshall KG: Prevention. How much harm? How much benefit? Physical, psychological and social harm. Can Med Assoc J 1996;155: 169-176.

3 Marteau MT: Psychology and screening. Narrowing the gap between efficacy and effectiveness. Br J Clin Psychol 1994;33:1-10.
4 Klock SC, Greenfeld DA: Psychological status of in vitro fertilization patients during pregnancy: A longitudinal study. Fertil Steril 2000;73: 1159-1164.

5 Koudstaal J, Braat DDM, Bruinse HW, Naaktgeboren N, Vermeiden JPW, Visser GHA: Obstetric outcome of singleton pregnancies after IVF: A matched control study in four Dutch university hospitals. Hum Reprod 2000;15: 1819-1825.
6 van der Ploeg HM: Handleiding bij de ZelfBeoordelings Vragenlijst, een Nederlandse bewerking van de Spielberger Stait-Trait Anxiety Inventory, STAI-DY, ed 2. Lisse, Swets en Zeitlinger, 2000.

7 Spielberger CD: Manual for the State-Trait Anxiety Inventory STAI (Form Y). Palo Alto, Consulting Psychologists Press, 1983.

8 Rose P, Humm E, Hey K, Jones L, Huson SM: Family history taking and genetic counselling in primary care. Fam Pract 1999;16:78-83. 